\title{
Supervision für helfende Berufe
}

\section{Astrid Schreyögg}

Online publiziert: 2. November 2015

(C) Springer Fachmedien Wiesbaden 2015

In diesem Heft geht es um die „Supervision für helfende Berufe“. Soeben ist das gleichnamige Buch von Nando Belardi (2015) in der 3., völlig überarbeiteten Neuauflage erschienen. Dieser Autor hatte schon 1992 dargelegt, dass die Supervisionsdebatte ursprünglich aus der Betreuung von Ehrenamtlichen resultierte, was dann in die Sozialarbeit mündete. Danach entwickelte sie sich in der Psychotherapie, um nachfolgend weitere Felder des sozialen Dienstleistungsbereichs zu erobern. Und in diesen Milieus ist sie bis heute unverzichtbar. 2008 attestierte ihr der Professionssoziologe Stefan Kühl ein hohes Professionalisierungsniveau „mit der Etablierung verbindlicher Ausbildungsstandards, der Organisation der in dem Feld Tätigen in Berufsverbänden und der Verpflichtung der Professionellen auf einen durchsetzbaren ethischen Berufskodex" (Kühl 2008, S. 125). Hier nennt der Autor besonders die großen Verbände, wie den Berufsverband Deutscher Psychologinnen und Psychologen und die Deutsche Gesellschaft für Supervision, die mit ihren Zertifikaten „Supervisor (BDP)“ und „Supervisor (DGSv)“"Qualitätssignale in den Markt gesandt haben. Daneben trugen auch kleinere Verbände wie die Deutsche Gesellschaft für Systemische Therapie und Familientherapie oder die Systemische Gesellschaft zu einer Marktschließung bei. Deshalb ist der potenzielle Wildwuchs in der Supervision helfender Berufe heute stark reduziert. Die Beratung der Mitglieder von Unternehmen und Verwaltungssystemen wird in Abgrenzung zur Supervision heute meistens "Coaching“ genannt. Bei Supervision handelt es sich um „Beratung der Beratung“, was dann als „Metaconsulting“ bezeichnet werden kann (Petzold 1998). In diesem Sinne wird vielfach auch die Arbeit von Coaches supervidiert.

Gleich im ersten Beitrag von Louis van Kessel geht es um eine viel praktizierte Form von Metaconsulting, nämlich um die Lehrsupervision. Dieser niederländische Autor beschreibt, wie die Lehrsupervision, d. h. die Supervision von Supervisoren, schon in den Ausbildungen von Supervisoren deren professionelles Verständnis und

Dr. A. Schreyögg $(\bowtie)$

Breisgauer Str. 29, 14129 Berlin, Deustchland

E-Mail: info@schreyoegg.de 
professionelles Handeln entwickeln und fördern kann. Die Lehrsupervision wird schon seit den 1970er Jahren von etlichen Ausbildungsinstituten laufend fortentwickelt und kann letztlich als ein wichtiger Indikator für die hohe Professionalität der Supervisionsdebatte dienen. Im zweiten Beitrag thematisieren Marie Drüge und Karin Schneider „Merkmale der Supervisionspraxis in der sozialen Arbeit“, dem „Ursprungsland der Supervision“. Die Autorinnen postulieren, dass die Supervision einen positiven Einfluss auf berufliche Kompetenz, Kooperation und sogar Entlastung hat. Es mangle allerdings bisher an entsprechenden empirischen beweisen. Aus diesem Grund haben sie 239 Beschäftigte der sozialen Arbeit anhand etlicher Kriterien nach dem Nutzen von Supervision befragt. Sie berichten positive Ergebnissen, betonen aber, dass unbedingt weiter geforscht werden sollte. Norbert Scholz befasst sich mit Konflikten in Teams und Organisationen. Er spricht von einem „Grenzgang zwischen Supervision, Mediation und Klärungshilfe“. Tatsächlich sind viele Teamprobleme nicht mit Formen der klassischen Supervision zu bearbeiten, denn dadurch kann eine Eskalation oft noch befördert werden. Für eine kompetente Bearbeitung empfiehlt er, einzelne Bausteine aus anderen Formaten in die Supervision zu integrieren. Der nachfolgende Beitrag von Sylvia Wagenaar beschäftigt sich mit Intervision. Dabei handelt es sich im Gegensatz zur Supervision um die leiterlose Auseinandersetzung mit der eigenen Praxis - hier mit der Praxis von Psychotherapie. Die Autorin hatte niedergelassene Psychotherapeuten befragt. Die Ergebnisse zeigen, welche äußeren Bedingungen und welche Ablauffaktoren entsprechend befriedigende Ergebnisse bringen.

Im Praxisbericht präsentiert uns Katharina Witte „Supervision mit Freiwilligen in sozialen Arbeitsfeldern“. Zwar hat sich die Supervision aus der Beratung von Volunteers entwickelt, sie haben aber meistens ganz andere Erwartungen als die Professionellen an den Supervisor. Sie möchten wie in den US-Amerikanischen Ursprüngen fachlich fortgebildet und geführt werden. Das hat auch seine Ursache darin, dass sie in der Organisation weniger verankert sind als die Hauptamtlichen.

In den beiden Diskursen fragen zunächst Laura Sedlarczek und Thomas Weber: „Ist eine Coaching-Kompetenz nützlich für Führungskräfte?“ Sie beantworten das letztlich mit ,ja“, denn diese Führungskräfte reagieren sensibler auf Komplikationen. Im zweiten Diskurs fragt Susanne Günther, ob Coaching in der Hochschuldidaktik doch nur eine didaktisch-methodische Beratung" ist. Sie zeigt, dass zwar 47 von 109 Hochschulen Coaching anbieten, die Begrifflichkeiten aber enorm divergieren. Sie meint, eine Homogenität in der Begriffsverwendung würde die Transparenz erheblich erhöhen.

\section{Literatur}

Belardi, N. (2015). Supervision für helfende Berufe (3., völlig überarb. u. aktual. Aufl.). Freiburg: Lambertus.

Kühl, S. (2008). Coaching und Supervision. Zur personenorientierten Beratung in Organisationen. Wiesbaden: VS Verlag.

Petzold, H. (1998). Integrative supervision, meta-consulting \& organisationsentwicklung. Paderborn: Junfermann. 